\title{
Recurrent patent infections with Toxocara canis in household dogs older than six months: a prospective study
}

\author{
Rolf Nijsse ${ }^{1 * \dagger}$, Lapo Mughini-Gras ${ }^{1,2 \dagger}$, Jaap A. Wagenaar ${ }^{1,3}$ and Harm W. Ploeger ${ }^{1}$
}

\begin{abstract}
Background: To reduce environmental contamination with Toxocara canis eggs, the current general advice is to deworm all dogs older than six months on average four times a year. However, only a small proportion of nonjuvenile household dogs actually shed T. canis eggs, and some dogs shed eggs more frequently than others. The identification of these frequent shedders and the associated risk factors is an important cornerstone for constructing evidence-based deworming regimens. The purpose of this study is to identify risk factors associated with recurrence of periods of shedding Toxocara eggs in a cohort of household dogs older than six months.

Methods: We performed a prospective study (July 2011 to October 2014) on shedding Toxocara eggs in a cohort of 938 household dogs older than six months from all over the Netherlands. The median follow-up time was 14 months. Monthly, owners sent faecal samples of their dogs for Toxocara testing and completed a questionnaire. Dogs were dewormed only after diagnosis of a patent infection (PI). Survival analysis was used to assess factors influencing the time to first diagnosed Pls (FPI) and the time to recurrent PIs (RPI).

Results: The overall prevalence of PIs was $4.5 \%$, resulting in an estimated average incidence of $0.54 \mathrm{PIs} / \mathrm{dog} / \mathrm{year}$. No PI was diagnosed in $67.9 \%$ of the dogs, $17.5 \%$ of the dogs went through only one PI and $14.6 \% \mathrm{had}>1 \mathrm{PI}$. Prevalence of Pls always peaked during wintertime. Increased hazards for first diagnosed Pls were associated with coprophagy, geophagy, walking off-leash for $\geq 80 \%$ of walking time, reported worms in the faeces, feeding a commercial diet and suffering from urologic or respiratory conditions. Median time to reinfection was nine months. Factors associated with increased hazards for recurrent Pls were taking corticosteroids, changing dog's main purpose, and proxies for veterinary care-seeking behaviours.

Conclusions: We concluded that targeted anthelmintic treatments in household dogs may be feasible as Pls tend to (re)occur in specific periods and in groups of dogs at high risk. Moreover, recurrent Pls appear to be influenced more by factors related to impaired immunity than environmental exposure to Toxocara eggs.
\end{abstract}

Keywords: Deworming, Dogs, Recurrent patent infections, Toxocara canis, Longitudinal study

\section{Background}

Toxocara canis is a worldwide-distributed parasitic roundworm of canids with recognized zoonotic potential [1-4]. In patent infections, adult T. canis worms live in the intestine of dogs and other canids, laying eggs that pass into the faeces and contaminate the environment

\footnotetext{
*Correspondence: e.r.nijsse@uu.nl

${ }^{\dagger}$ Equal contributors

'Department of Infectious Diseases and Immunology, Faculty of Veterinary Medicine, Utrecht University, P.O. box 80.165, 3508, TD, Utrecht, The Netherlands

Full list of author information is available at the end of the article
}

[5]. Within these eggs, a third-stage larva develops, after which the eggs are infective. This embryonation process usually takes several weeks [6, 7]. Like other paratenic hosts, humans can become infected by ingesting embryonated eggs or larvae in raw or undercooked meat.

In young dogs ( $\leq$ six months of age), the ingestion of infective T. canis eggs is most likely to lead to hepatotracheal migration of the larvae followed by a patent infection. Conversely, the ingestion of infective eggs by older dogs (> six months of age) is less likely to lead to patent infections, as dogs develop immunity against the 
tracheal migration of the larvae $[8,9]$, resulting in so-called somatic migration [10]. This migration route leads to larvae residing somewhere in a dog's body where they can survive for long periods, but it does not lead to a patent infection. Therefore, most dogs older than six months do not actively contribute to the environmental contamination with $T$. canis eggs. Yet, some dogs older than six months do occasionally develop patent $T$. canis infections [11]. This is likely due to insufficient levels of built-up immunity or to temporary changes in immunity, e.g. because of endocrinologic perturbations, immune disorders or stress. Also the uptake of low numbers of infective eggs $[9,11,12]$ or the infection with larvae (rather than infective eggs) by consumption of raw meat and offal from infected paratenic hosts can lead to patent infections in adult dogs due to the evasion or avoidance of acquired immunity on lung level [10].

The fact that a few dogs older than six months do shed T. canis eggs [13-16], posing a risk for human infection, is used to justify the current 'preventive' three-to-four-timesa-year blind deworming advice for household dogs in this age category [17]. However, it has not yet been proved that such a treatment strategy is effective in reducing the contamination of the environment $[16,18]$, whilst it does lead to numerous treatments administered in absence of an actual patent infection to be treated. Therefore, monthly or three-monthly faecal examinations are also recommended as a feasible alternative to 'preventive blind treatment' [17]. To implement evidence-based treatment strategies for dogs, it is crucial to identify dogs that are prone to develop patent $T$. canis infections [13, 19-21]. In young dogs or in dogs infected with larvae instead of eggs, a defined prepatent period can be used for preventive treatment. For most other dogs, however, a suitable interval is less obvious because the acquired immunity will prevent the development of patent infections or prolong the prepatent period to variable extents following ingestion of infective eggs. Crosssectional studies in North-European countries show that, at any given point in time, about $5 \%$ of household dogs shed T. canis eggs in their feces [14-16, 22, 23]. However, such studies usually fail to show to what extent and at what interval dogs older than six months of age experience recurrent T. canis infections. Adult dogs that are frequent egg shedders are more suited targets for regular treatments. To address the occurrence of recurrent $T$. canis infections in non-juvenile dogs, we performed a longitudinal study comprising a large cohort of household dogs older than six months in the Netherlands. The aim of this study was to determine the frequency of, and factors associated with, recurrent patent $T$. canis infections in these dogs.

\section{Methods}

\section{Study design and dog population}

Each month for a maximum period of 40 months (July 2011 to October 2014), dog owners in the Netherlands were asked to submit a faecal sample of their $\operatorname{dog}(\mathrm{s})$ to be examined for the presence of helminth eggs (see below) at the faculty of Veterinary Medicine of Utrecht University. Along with each submitted sample, owners were asked to complete a web-based questionnaire to collect relevant epidemiological information (see below). Dog owners were enrolled via advertising the opportunity of enrolment in the study across pet shops, veterinary clinics, pet-themed websites and dog breed societies in the Netherlands. Additionally, flyers were handed out at some dog walking areas. Recruitment of dogs from already participating owners was allowed during the entire study period. To be enrolled in the study, dogs had to be at least six months of age and, for logistic reasons, each owner was allowed to enrol a maximum of four dogs. Laboratory results were sent monthly by e-mail to the participating dog owners. Once enrolled in the study, dogs were not allowed to be dewormed unless a positive laboratory result was obtained, the dogs were traveling to Dirofilaria immitis-endemic areas, or they were lactating and performing litter care. In case of a positive laboratory result, the owners were asked to prevent their dog from eating anything from the ground for at least 3 days and send in a new sample. This step was included to rule out positive samples due to coprophagy as much as possible [24, 25]. If this confirmation sample tested positive also, it was considered a patent infection. After a positive confirmation sample, a short-acting anthelminthic product (containing febantel, pyrantel and praziquantel) was provided. If a parasitic infection (e.g. Cystoisospora spp.) was diagnosed that could not, either legally or due to suboptimal efficacy, be cured with this anthelminthic, owners were advised to confer with their veterinarian.

Owners participated in this study knowing that the acquired data would be used for a scientific publication.

\section{Collection of epidemiological data}

Epidemiological data were collected via a self-administered questionnaire that could be answered online. We differentiated between the starting questionnaire (completed at submission of the first faecal sample) and the follow-up questionnaires, which were completed at submission of each subsequent sample. The starting questionnaire contained questions about the dog's age, sex, breed, function, reproductive status, living conditions, diet, time roaming freely, predatory and coprophagic behavior, health status, medication use, and deworming history. The follow-up questionnaires were meant to monitor any change in living conditions, lifestyle (e.g. diet, function, etc.) or health of the dogs relative to the preceding questionnaire. Owners were specifically asked to report whether and when their dogs had been dewormed for reasons other than those provided above. A copy of the questionnaires is available as supplementary data (Additional file 1). Information on 
socio-economic status (SES, a normalized score ranging from - 4 to +4 based on income, employment and educational level per postcode area) and urbanization degree $(>2,000,1,500-2,000,1,000-1,500,500-1,000$, and $<500$ addresses $/ \mathrm{km} 2$ ) was obtained at the postal code level from Statistics Netherlands (http://www.cbs.nl/en-GB/ menu/home/default.htm).

\section{Coproscopical examination}

Samples were submitted individually from each dog using a collection box at the faculty of Veterinary Medicine of Utrecht University (for people living or working close by) or submitted to the laboratory by regular mail, using study-provided materials and instructions. Each sample was identified by a unique code, which was linked to the questionnaire. The centrifugal sedimentation and flotation technique used for coproscopical analysis has been reported previously $[16,25,26]$. For each sample, at least three grams of faeces was used and a sugar solution (s.g. $1.27-1.30 \mathrm{~g} / \mathrm{cm}^{3}$ ) was used as flotation medium. This method has a theoretical detection limit of detecting 1.6 eggs per gram. Slides were microscopically examined at $40 \times, 100 \times$ and $400 \times$ magnification. T. canis eggs were measured and morphologically identified, using the AAVP reference guide for diagnosing parasitism in animals [27]. For logistic reasons, two samples (three grams each) were pooled in the laboratory for first testing, with a theoretical detection limit of 3.2 eggs per gram for each individual dog in the pooled sample. If this pooled sample tested positive for dog-typical parasites, the samples were re-tested separately to determine which sample contained the eggs.

\section{Data analysis}

Survival analysis was used to assess factors influencing the time to the "first" diagnosed event of Toxocara egg shedding (first patent infection $=$ FPI) and the time to recurrence of a patent infection (recurrent patent infection $=$ RPI) in our dog population. This was done using Cox proportional hazards models, which assessed the risk of patent Toxocara (re)infection longitudinally as a function of the factors measured at each sampling event. For the time to FPI, dogs entered the cohort at the submission of the first sample and were censored at their first diagnosed infection. Observation time for the time to FPI was then calculated as the time from the submission of the first sample (i.e. enrolment in the study) to that of the FPI or the end of the follow-up period (i.e. end of study or dropout from study). For the time to RPI, entry into the cohort began with the FPI and dogs were not censored after each subsequent reinfection. A conditional risk set model [28], in which the analysis is stratified by event (i.e. infection) order, was used for the analysis of the time to RPI. The assumption is that the conditional risk at time $t$ for event $k$ derives from all subjects under observation at time $t$ that have had event $k-1$. The method is widely used for analysis of recurrent events in the biomedical literature [29]. Observation time for the time to RPI was then defined as the gap time between subsequent infections (i.e. time to each event is measured from the previous event), or from the FPI to the end of the follow-up period (end of study or dropout from study) if the dogs did not have a RPI. Associations were expressed as hazard ratios (HRs) with $95 \%$ confidence interval $(95 \% \mathrm{CI})$.

Preliminary analyses included log-rank tests for equality of survivor functions and Kaplan-Meier curves to assess graphically the assumption of proportionality for Cox proportional hazards for each independent variable. Variables satisfying these conditions were selected for inclusion in a multivariable Cox proportional hazard regression model. A backward stepwise selection procedure was then applied, with variables showing a $P \leq 0.05$ for the association with the outcome variable being retained in the model. The effect of removing variables on the associations of the other covariates was also monitored. A change of $\geq 10 \%$ in the coefficients was considered as a sign of confounding and the variable in question was retained in the model regardless of significance. The variables dog's age (6-12 months, $1-7$ years, $>7$ years), sex, season (winter, December-February; autumn, September-November; spring, March-May; summer, June-August), time since last deworming (continuous variable expressed in months) and reported coprophagic behaviour were always controlled for in the models. The tested variables are intrinsic to the questions in the questionnaire (Additional file 1). The SES was included as test variable, obtained at postcode level. Biologically plausible interactions between covariates were also assessed and the final model was expanded to include significant interaction terms, if any. Besides the repeated measurements made on the same dogs over time (multiple-record-per-subject analysis), we accounted for clustering (or non-independence) of dogs living in the same household (i.e. having the same owner) by incorporating cluster-robust variance estimators. Statistical analysis was performed using STATA 13 (StataCorp LP, College Station, USA).

\section{Results}

\section{Descriptive statistics}

In total, 938 dogs belonging to 570 owners were enrolled in the study. The cohort was followed for a total of 12,968 dog-months. Figure 1 shows the distribution of dogs over the number of months of follow-up. The median followup time per dog was 14 months (interquartile range [IQR] 5-22 months). The median age of the dogs at enrolment was 4 years (IQR $2-7$ years). The study population 


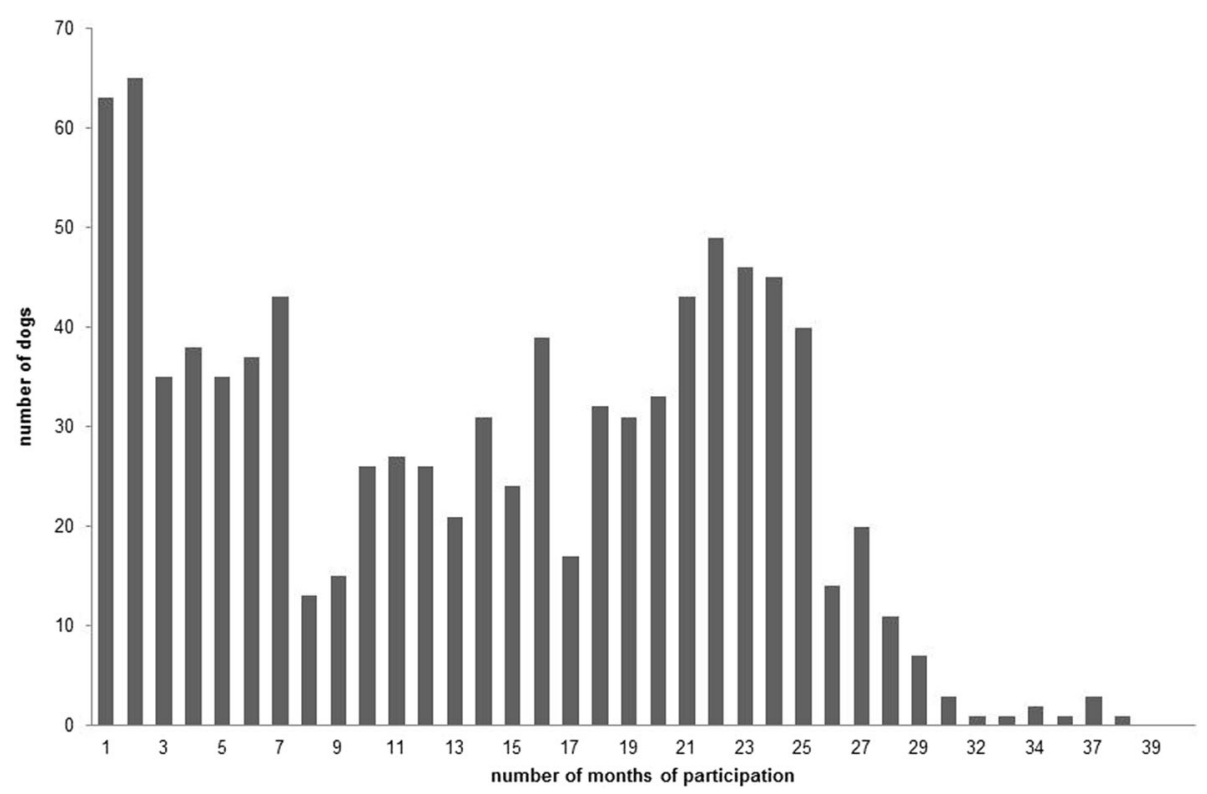

Fig. 1 Distribution of dogs over duration of follow up. The distribution of duration of participation in months with the corresponding number of dogs

consisted of 406 (43.3 \%) males and 532 (56.7 \%) females ( male/female ratio $=0.76$ ).

Of 12,968 stool samples tested, 585 were positive for Toxocara eggs, resulting in an overall proportion of $4.5 \%$ (95\% CI 4.0-5.1\%) positive samples. Table 1 shows the number of dogs and corresponding number of samples stratified by their number of positive test months diagnosed during the study period. In total, 301 (32.1 \%) dogs had at least one Toxocara infection, whereas the remaining 637 dogs $(67.9 \%)$ never tested positive. The incidence rate was estimated at 0.54 patent Toxocara infections (95\% CI 0.48-0.61) on average per dog/year. Anthelmintic treatment was given in 84 occasions for reasons unrelated to the study (e.g. foreign travel), in these cases dogs were allowed to continue their enrollment in the project.
The monthly Toxocara incidence rate showed a clear seasonal pattern (Fig. 2), peaking during the winter and decreasing during the summer. Figure 2 also shows a decreasing trend in the incidence over the years.

\section{Survival analysis}

\section{Time to "first" infection}

Survival analysis for the time to FPI was based on 836 dogs with observations not ending on entry or beginning on FPI. These dogs accounted for a total of 8,783 dogmonths at risk under observation during which 259 FPI occurred, resulting in an incidence rate of 2.9 FPIs per 100 dog-months (95\% CI 2.6-3.3). Median time to FPI was 5 months (IQR 2-10).

Table 1 Dogs and samples stratified by number of Toxocara eggs positive test months

\begin{tabular}{|c|c|c|c|c|c|}
\hline \multirow{2}{*}{$\begin{array}{l}\text { Number of patent } \\
\text { infections }\end{array}$} & \multirow{2}{*}{$\begin{array}{l}\text { Number of } \\
\text { dogs (\%) }\end{array}$} & \multicolumn{4}{|c|}{ Samples } \\
\hline & & $n$ & $\begin{array}{l}\text { Toxocara } \\
\text { negative }\end{array}$ & $\begin{array}{l}\text { Toxocara } \\
\text { positive }\end{array}$ & $\begin{array}{l}\text { Mean number of } \\
\text { samples per dog }\end{array}$ \\
\hline 0 & $637(67.9)$ & 7,706 & 7,706 & 0 & 12 \\
\hline 1 & 164 (17.5) & 2,761 & 2,597 & 164 & 17 \\
\hline 2 & $66(7.0)$ & 1,188 & 1,056 & 132 & 18 \\
\hline 3 & $33(3.5)$ & 566 & 467 & 99 & 17 \\
\hline 4 & $18(1.9)$ & 347 & 275 & 72 & 19 \\
\hline 5 & $9(1.0)$ & 174 & 129 & 45 & 19 \\
\hline 6 & $8(0.9)$ & 164 & 116 & 48 & 21 \\
\hline 8 & $2(0.2)$ & 38 & 22 & 16 & 19 \\
\hline 9 & $1(0.1)$ & 24 & 15 & 9 & 24 \\
\hline Total & $938(100)$ & 12,968 & 12,383 & 585 & \\
\hline
\end{tabular}




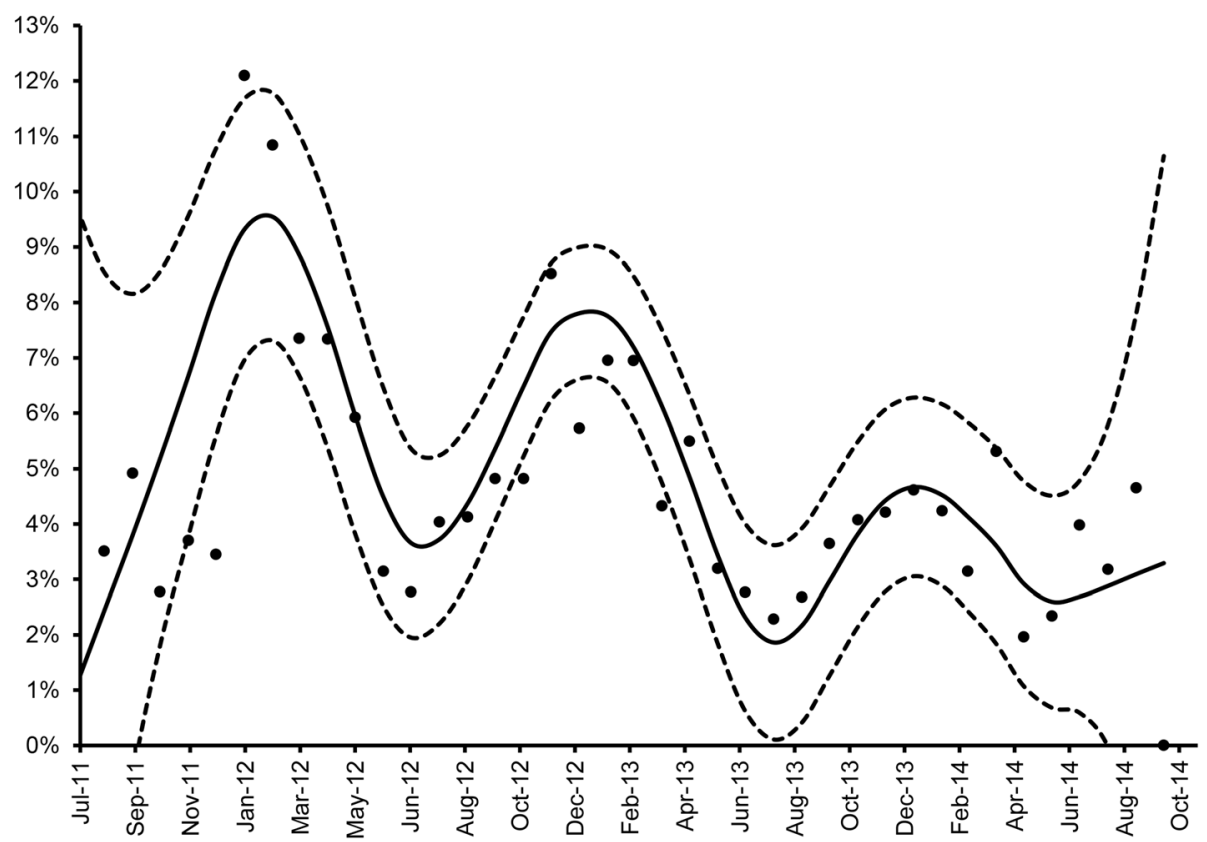

Fig. 2 Monthly T. canis incidence (dots) over the study period (from July 2011 to October 2014). An optimized cubic smoothing P-spline function (solid line) and corresponding $95 \%$ confidence interval (dotted lines) is fitted to the observed data

The final multivariable Cox proportional hazards model for Toxocara FPI (Table 2) showed that the risk of observing a FPI was higher for dogs displaying coprophagic behavior or eating sand/soil, dogs ranging off-leash $>80 \%$ of their walking time as compared to dogs ranging freely $\leq 20 \%$ of their walking time, dogs whose owners had noticed worms in their dogs' faeces, dogs fed with a commercial diet and dogs with urologic or respiratory conditions. The risk of having a FPI was also significantly higher in winter and autumn as compared to summer, and it increased with increasing time since last deworming. Conversely, older age groups, having neurologic conditions, and being fed with a diet containing frozen raw meat had a lower risk.

\section{Time to reinfection}

Time to RPI analysis was based on 281 dogs in which a FPI was diagnosed and from which subsequent samples were submitted. The corresponding incidence rate of 284 reinfections over 3,247 dog-months at risk under observation was 8.7 RPIs per 100 dog-months (95\% CI 7.7-9.7). Median time to RPI was 9 months (IQR 3-16).

The multivariable Cox proportional hazards regression model for Toxocara reinfection (Table 3) showed that the risk of reinfection was significantly higher for dogs receiving corticosteroid treatment, for dogs whose main purpose/use was changed, and for dogs whose owners reported that they would usually buy anthelmintic drugs at veterinary clinics. The risk of reinfection was also significantly higher in winter as compared to the summer, and it increased with increasing time since last deworming. Conversely, the risk of RPI was significantly lower for dogs whose owners reported to sometimes or always collect and dispose of their dogs' faeces as compared to those who reported to never do that, as well as for dogs with neurologic conditions, and was borderline significant for dogs with orthopedic conditions.

\section{Discussion}

Longitudinal studies are better suited than cross-sectional studies to investigate events that can recur throughout an individual's life. Taking the limitations of coproscopical examination to diagnose patent Toxocara infections into account [30], our study reports an estimated prevalence of $4.5 \%$ dogs that shed Toxocara eggs, which is comparable with reported, mostly cross-sectional, prevalences from current literature [14-16, 22, 23]. Monthly incidence ranged from 2 to $12 \%$, peaking consistently during wintertime in all three years of follow-up. This finding was unexpected, as one would hypothesize that in a country like the Netherlands where seasons are well defined, dogs are walked outdoor for longer periods (and perhaps more often unleashed) during the summer as compared to winter because of the generally more favorable/pleasant weather conditions, and this would impose a higher risk for infection. Yet, similar seasonal patterns were noted by others $[15,31,32]$. Although no exhaustive explanation can be provided, it is evident that the winter peaks were consistently present in all three years of follow-up. The possibility that the observed seasonal 
Table 2 Results of the final multivariable Cox proportional hazards regression model for "first" T. canis infection

\begin{tabular}{|c|c|c|c|c|c|c|c|}
\hline \multirow{2}{*}{ Age group } & \multirow[t]{2}{*}{ No. of dogs } & \multirow[t]{2}{*}{ No. of dog-months at risk } & \multirow[t]{2}{*}{ No. of observed FPIs } & \multirow[t]{2}{*}{ HR } & \multicolumn{2}{|c|}{$95 \% \mathrm{Cl}$} & \multirow[t]{2}{*}{$P$-value } \\
\hline & & & & & & & \\
\hline 0-12 months & 202 & 636 & 42 & Ref. & & & \\
\hline $1-7$ years & 575 & 5,678 & 157 & 0.47 & 0.32 & 0.67 & $<0.0001$ \\
\hline$>7$ years & 250 & 2,469 & 60 & 0.40 & 0.26 & 0.61 & $<0.0001$ \\
\hline \multicolumn{8}{|l|}{ Sex } \\
\hline Male & 362 & 3,702 & 113 & Ref. & & & \\
\hline Female & 481 & 5,081 & 146 & 0.93 & 0.72 & 1.20 & 0.580 \\
\hline \multicolumn{8}{|l|}{ Coprophagy } \\
\hline No & 475 & 4,877 & 115 & Ref. & & & \\
\hline Yes & 400 & 3,906 & 144 & 1.36 & 1.05 & 1.77 & 0.021 \\
\hline \multicolumn{8}{|l|}{ Sampling season } \\
\hline Summer & 655 & 2,332 & 38 & Ref. & & & \\
\hline Winter & 741 & 2,434 & 89 & 1.73 & 1.14 & 2.61 & 0.009 \\
\hline Autumn & 564 & 1,978 & 76 & 1.62 & 1.05 & 2.50 & 0.030 \\
\hline Spring & 603 & 2,039 & 56 & 1.28 & 0.81 & 2.01 & 0.287 \\
\hline \multicolumn{8}{|l|}{ Eating soil/sand } \\
\hline No & 740 & 7,818 & 213 & Ref. & & & \\
\hline Yes & 106 & 965 & 46 & 1.62 & 1.12 & 2.35 & 0.011 \\
\hline \multicolumn{8}{|l|}{ Following a commercial diet } \\
\hline No & 320 & 3,081 & 68 & Ref. & & & \\
\hline Yes & 567 & 5,702 & 191 & 1.47 & 1.08 & 2.00 & 0.014 \\
\hline \multicolumn{8}{|l|}{$\begin{array}{l}\text { Following a diet containing } \\
\text { frozen raw meat }\end{array}$} \\
\hline No & 381 & 3,377 & 131 & Ref. & & & \\
\hline Yes & 494 & 5,406 & 128 & 0.68 & 0.52 & 0.89 & 0.005 \\
\hline \multicolumn{8}{|l|}{ Having respiratory conditions } \\
\hline No & 822 & 8,456 & 244 & Ref. & & & \\
\hline Yes & 46 & 327 & 15 & 1.84 & 1.08 & 3.13 & 0.026 \\
\hline \multicolumn{8}{|l|}{ Having neurologic conditions } \\
\hline No & 819 & 8,413 & 257 & Ref. & & & \\
\hline Yes & 40 & 370 & 2 & 0.21 & 0.06 & 0.82 & 0.024 \\
\hline \multicolumn{8}{|l|}{ Having urologic conditions } \\
\hline No & 816 & 8,467 & 245 & Ref. & & & \\
\hline Yes & 41 & 316 & 14 & 1.79 & 1.12 & 2.86 & 0.015 \\
\hline \multicolumn{8}{|l|}{ Excreting worms in faeces } \\
\hline No & 828 & 8,688 & 252 & Ref. & & & \\
\hline Yes & 10 & 95 & 6 & 2.26 & 1.16 & 4.42 & 0.017 \\
\hline \multicolumn{8}{|l|}{ Off-leash walking time (\%) } \\
\hline$\leq 20$ & 131 & 1,325 & 29 & Ref. & & & \\
\hline $20-50$ & 260 & 2,461 & 54 & 1.06 & 0.64 & 1.75 & 0.829 \\
\hline $50-80$ & 141 & 1,337 & 40 & 1.30 & 0.76 & 2.23 & 0.341 \\
\hline$>80$ & 375 & 3,660 & 136 & 1.79 & 1.13 & 2.83 & 0.013 \\
\hline Time since last deworming (months) ${ }^{a}$ & - & - & - & 1.002 & 1.000 & 1.003 & 0.024 \\
\hline
\end{tabular}

${ }^{a}$ Continuously time-varying variable let interact with the underlying time variable 
Table 3 Results of the final multivariable Cox proportional hazards regression model for T. canis reinfection

\begin{tabular}{|c|c|c|c|c|c|c|c|}
\hline & No. of dogs & No. of dog-months at risk & No. of observed RPIs & $\mathrm{HR}$ & \multicolumn{2}{|c|}{$95 \% \mathrm{Cl}$} & $P$-value \\
\hline \multicolumn{8}{|l|}{ Age group } \\
\hline 6-12 months & 49 & 110 & 30 & Ref. & & & \\
\hline $1-7$ years & 202 & 2,211 & 170 & 0.85 & 0.49 & 1.46 & 0.552 \\
\hline$>7$ years & 96 & 926 & 84 & 0.87 & 0.47 & 1.62 & 0.663 \\
\hline \multicolumn{8}{|l|}{ Sex } \\
\hline Male & 121 & 1,400 & 125 & Ref. & & & \\
\hline Female & 162 & 1,847 & 159 & 1.27 & 0.91 & 1.77 & 0.160 \\
\hline \multicolumn{8}{|l|}{ Coprophagy } \\
\hline No & 118 & 1,234 & 84 & Ref. & & & \\
\hline Yes & 170 & 2,013 & 200 & 1.09 & 0.74 & 1.59 & 0.674 \\
\hline \multicolumn{8}{|l|}{ Sampling season } \\
\hline Summer & 237 & 777 & 57 & Ref. & & & \\
\hline Winter & 220 & 612 & 95 & 1.68 & 1.15 & 2.46 & 0.008 \\
\hline Autumn & 239 & 857 & 50 & 1.30 & 0.82 & 2.08 & 0.266 \\
\hline Spring & 244 & 1,001 & 82 & 1.43 & 0.96 & 2.14 & 0.079 \\
\hline \multicolumn{8}{|l|}{ Taking corticosteroids } \\
\hline No & 265 & 3,036 & 263 & Ref. & & & \\
\hline Yes & 25 & 211 & 21 & 2.38 & 1.09 & 5.19 & 0.029 \\
\hline \multicolumn{8}{|l|}{$\begin{array}{l}\text { Frequency of dog's faeces } \\
\text { removal/disposal }\end{array}$} \\
\hline Never & 32 & 351 & 48 & Ref. & & & \\
\hline Sometimes & 160 & 1,903 & 164 & 0.54 & 0.33 & 0.86 & 0.01 \\
\hline Always & 89 & 993 & 72 & 0.54 & 0.32 & 0.91 & 0.02 \\
\hline \multicolumn{8}{|l|}{ Change in dog's main purpose/use } \\
\hline No & 280 & 3,238 & 281 & Ref. & & & \\
\hline Yes & 8 & 9 & 3 & 10.84 & 1.14 & 103.21 & 0.038 \\
\hline \multicolumn{8}{|l|}{ Having neurologic conditions } \\
\hline No & 279 & 3,188 & 283 & Ref. & & & \\
\hline Yes & 9 & 59 & 1 & 0.11 & 0.02 & 0.74 & 0.023 \\
\hline \multicolumn{8}{|l|}{ Having orthopaedic conditions } \\
\hline No & 262 & 2,891 & 260 & Ref. & & & \\
\hline Yes & 45 & 356 & 24 & 0.55 & 0.28 & 1.06 & 0.074 \\
\hline \multicolumn{8}{|l|}{$\begin{array}{l}\text { Owner usually buys anthelmintic } \\
\text { drugs at veterinary clinics }\end{array}$} \\
\hline No & 134 & 1,464 & 107 & Ref. & & & \\
\hline Yes & 147 & 1,783 & 177 & 1.52 & 1.10 & 2.11 & 0.011 \\
\hline Time since last deworming (months) ${ }^{a}$ & - & - & - & 1.003 & 1.001 & 1.005 & $<0.0001$ \\
\hline
\end{tabular}

${ }^{a}$ Continuously time-varying variable let interact with the underlying time variable

Abbreviations: HR hazard ratio; $95 \% \mathrm{Cl}, 95 \%$ confidence interval; RPI recurrent patent infection; Ref. reference category

pattern reflects more frequent deworming in summertime could be ruled out because the surveyed dog population was not routinely dewormed, as this was a condition for participation in the study. Wolves (Canis lupus), the far ancestors of dogs, are mono-estrus species that breed in mid to late winter, and the associated endocrinological changes might reactivate dormant $T$. canis larvae during that period as is known in dogs. It is likely that the change in day length is the stimulus of this breeding cycle. We speculate that in the co-evolution of the parasite and its definitive host, this phenomenon might have persisted even though household dogs do not necessarily show a well-defined seasonal breeding pattern any longer [33]. However, kenneled cyclic beagles were not at higher risk 
for developing a patent $T$. canis infection, which makes this hypothesis less likely [34]. An additional explanation might be that shorter walks and staying longer at home during wintertime may act as stressor, contributing to reactivation of dormant larvae. Reactivation of dormant somatic larvae is likely to be responsible instead of an increased risk of being re-infected by ingestion of infective eggs. Another possible stressor could be related to the intensive use of fireworks during the festive period in the Netherlands in the last months of the year. A recent study reported increased cortisol levels, a common indicator of stress, in dogs during winter [35]. Whatever the reasons might be, it is apparent that seasonality in T. canis egg shedding exists and needs to be considered in future studies, especially when these are performed cross-sectionally at one moment in time. Moreover, understanding the origin of this seasonal pattern is relevant for control, as any (blind) deworming would be more likely to be necessary during the coldest rather than the warmest months. Besides seasonality, monthly Toxocara incidence also tended to decline over time even though no blind deworming was applied. This may be explained by the aging of the cohort and by the loss of follow-up of some frequent shedders.

Most (67.9\%) participating dogs were never diagnosed with a patent $T$. canis infection during the follow-up period, $17.5 \%$ dogs experienced only one infection, and $14.6 \%$ dogs experienced two or more infections, with a maximum of nine patent infections diagnosed in the same dog during a follow-up period of 24 months. Based on the observed frequency of infection, the average annual incidence rate was estimated at 0.54 patent infections/dog/year, which can be translated into one infection occurring approximately every two years among household dogs that are not (blindly) treated on a regular basis. Consequently, it could be said that the currently propagated four-times-a-year anthelmintic treatment advice lacks evidence for dogs older than six months. Our data suggest that targeted treatments may be preferable over blind treatments. A two-step approach was applied in the longitudinal analysis. First, a survival analysis for identifying factors influencing the time to FPI was performed. Secondly, survival analysis was performed for identifying factors influencing the time to RPI. The time to FPI was measured from the moment of enrollment till the first diagnosed patent infection, without knowing when these dogs had actually experienced the previous patent infection before participating in the study. In general, similar risk factors for FPI were found in the present study compared to previous (cross-sectional) studies [13,19-21], as well as to a previous cross-sectional study based on the same dog population, where only the first submitted sample of a dog was included, as in the present study [16]. These were young age, coprophagy, and proportion of walking time walking off-leash. An unexpected risk factor was the feeding of a commercial diet, while feeding frozen raw meat appeared to be protective. Though most of the $T$. canis larvae present in the meat will be killed by freezing it, this is not always the case [36-38]. The assumption that raw meat may be a risk factor for $T$. canis infection is only valid if the meat in question contains dormant larvae. The origin of the consumed meat is therefore important to take into account, as meat from farms with a high level of biosecurity is highly unlikely to contain dormant larvae. Yet, previous research indicated that owners feeding raw meat to their dogs do not often know the origin of that meat (unpublished data). Explaining that commercial (bagged/canned) diets are a risk factor for patent $T$. canis infections is difficult. It is important to realize that this association might just be a spurious one due to a hitherto unknown confounder that was not accounted for in the analysis. We speculate that dogs receiving a commercial diet, which is usually easier for dogs to eat, might be more prone to the need of chewing/gnawing items, perhaps from the ground outside increasing the risk of infection. However, estimates were adjusted for "eating soil/sand" (included as covariate in the model) and were not influenced by the factor "eating items from the ground" (not significant). Interestingly, in the analysis of recurrent infections, factors related to diet were not associated with testing positive on Toxocara eggs. Eating soil/sand turned out to be a risk factor for FPI. This suggests that infective eggs are ingested, as coprophagy was controlled for in the analysis, or that eggs passively pass the gastro-intestinal tract after eating soil/sand. Normally, this would not lead to patent infection in adult dogs. However, it has been reported that infection with low numbers of eggs may sometimes lead to patent infection, as low numbers of larvae may pass undetected by the host's immune system during their hepatic-tracheal migration [12]. The observed effects of some health conditions on the risk of Toxocara egg shedding may be a reflection of the stress induced by the conditions themselves and/or by the decreased immune-competence that these conditions may entail. In contrast, having neurologic or orthopedic conditions stood out as a protective factor. Dogs with these conditions tend to be less active outside, thereby reducing the risk of acquiring a $T$. canis infection from the environment, which may oppose an effect of stress induced by these conditions.

The analysis of RPIs showed an incidence of 8.7 reinfections/100 dog-months, more than three times that for FPIs. This suggests that recurrent shedding of Toxocara eggs occurs more often in some dogs that for some reason are particularly prone to experience multiple patent T. canis infections. Such dogs may be called "wormy" dogs and, hence, should be a specific target for 
treatments. This group of "wormy" dogs is responsible for the majority (421 of 585 or $72 \%$ ) of positive faeces samples (see Table 1).

Determining factors associated with (recurrent) infections in these dogs would therefore provide useful targets for control. The factors associated with RPIs found here showed some overlap with those for the FPIs. However, there were also some interesting differences, which mainly concerned factors mirroring the immunological status of the dog. For instance, the administration of corticosteroids, known for their immunosuppressive action, resulted in a HR of 2.38 for experiencing a RPI. Sudden changes in the routine of the dog (i.e. main purpose or use of the dog), which may well lead to a temporarily suboptimal immune status due to stress, resulted in a hazard ratio of 10.84 . The latter becomes even more plausible when having a closer look at the data (results not shown), as the owners whose dogs had their purpose changed mostly reported that their dogs had become hunting dogs. It is known that hunting activities can be quite stressful for dogs [39]. Previously identified risk factors for patent Toxocara infection may also be explained, to some extent, by (temporary) perturbations of the immune status, such as being kenneled [16]. This implies that dogs under periods of stress are at risk of becoming shedders of Toxocara eggs and should be targeted by anthelmintic treatment.

Cleaning up dogs' faeces by owners appeared to be protective for RPIs. Although such behavior in a dog owner is unlikely to be directly related to the risk of infection in the respective dog, it may mirror a general habit of disposing of dogs' faeces in the area where the owner lives, and therefore to a societal pressure to clean up dogs' faeces, possibly resulting in a generally less contaminated environment with $T$. canis eggs shed by dogs. Coprophagy was identified as a risk factor for FPI, as well as in a previous cross-sectional study [16], but it was no longer significant for RPIs. This suggests that $T$. canis eggs in the faeces of dogs showing recurrent infections are more likely to be eggs from an actual infection rather than eggs simply passing the gastrointestinal tract after ingestion of unembryonated eggs with the faeces of real $T$. canis shedders. In contrast, dogs incidentally shedding Toxocara eggs may often do so because of coprophagy. Coprophagy is a possible factor that can influence the outcome of coproscopical examinations and when performing such methods it should be considered when an animal tests positive $[24,25]$. Finally, buying anthelmintics at the veterinary clinic was a risk factor for RPIs. This is hard to explain by simply looking at the biology of the parasite or the host. However, because anthelmintics in the Netherlands can also be purchased (sometimes for cheaper prices) at pet stores, internet, supermarkets, and department stores, owners buying anthelmintics at veterinary clinics do so probably because they happen to frequently visit the clinic for the health problems of their dogs, so this factor may simply mirror frequent veterinary care-seeking behaviors because of impaired health in the dogs.

\section{Conclusions}

Following a large cohort of dogs, all older than six months, up to three years without performing routine deworming in absence of a confirmed diagnosis revealed that approximately $68 \%$ of dogs never tested positive for Toxocara eggs. The overall incidence rate was 0.54 patent Toxocara infections/dog/year, meaning that a nonroutinely treated dog is likely to shed Toxocara eggs once every two years, on average. However, the incidence rate of RPIs was much higher than that of FPIs, suggesting that there is a group of dogs particularly prone to recurrence of patent Toxocara infections. Dogs with RPI were responsible for the majority of positive faeces samples.

The identified risk factors for FPIs and RPIs indicate that there are two important aspects to consider when assessing the risk for a dog to acquire a Toxocara infection, the exposure to sources of infection and the failure of immunity. Indeed, both the likelihood of ingesting infective eggs/larvae and the possible evasion of immunity, perhaps by already present somatic larvae, should be taken into account when controlling $T$. canis infections in household dogs. Based on our study, this can be indicated by factors related to immune suppression, e.g. administration of immunosuppressive drugs or stress caused by underlying diseases or changes in routine, as well as factors related to higher chances of ingesting $T$. canis eggs from the environment, e.g. eating soil/sand or enjoying a high amount of off-leash walking time. Future modelling papers may benefit from studies that report on risk factors, especially when studied in a longitudinal set-up, so different scenarios can be tested by varying the exposure to different factors over time. Together with the observed peaks of Toxocara incidence during the winter months, our results suggest that blind deworming may be refined to become a more targeted deworming strategy based on the identified risk factors.

\section{Additional file}

Additional file 1: Questionnaire. (PDF $483 \mathrm{~kb}$ )

\section{Abbreviations}

PI: Patent infection; FPI: First diagnosed patent infection; RPI: Recurrent patent infection

\section{Acknowledgements}

The authors thank Bayer Animal Health for partially financing this study. Bayer Animal Health did not have any influence on the design of this study or on the outcomes. The Veterinary Microbiological Diagnostic Centre of the Faculty of Veterinary Medicine of Utrecht University, Stephanie Rodenberg 
and students performing their research internship are acknowledged for their substantial contribution to the laboratory work and Nicole Buijtendijk Charlotte de Wolf and Erwin Virginia for the administrative support. The owners participating in this study are acknowledged for supplying faeces and answering the questionnaires.

\section{Funding}

Non applicable.

\section{Availability of data and materials}

The datasets generated during and/or analyzed during the current study are not publicly available due to reasons concerning the privacy of the participating owners.

\section{Authors' contributions}

RN and LM were involved in designing the study, analyzing the results, interpretation of the outcomes, and writing and revising the manuscript. RN was involved in coordinating the study and generating data concerning dogs and the questionnaires. LM was involved in constructing the survival analysis. LM and RN contributed equally in this paper. JW and HP were involved in designing the study, critically revising the manuscript and in the interpretation of results. All authors read and approved the final manuscript.

\section{Competing interests}

The authors declare that they have no competing interests.

\section{Consent for publication}

All dog owners voluntarily participated in this study and agreed on publication of the anonymized data.

\section{Ethics approval and consent to participate} Non applicable.

\section{Author details}

Department of Infectious Diseases and Immunology, Faculty of Veterinary Medicine, Utrecht University, P.O. box 80.165, 3508, TD, Utrecht, The Netherlands. ${ }^{2}$ National Institute for Public Health and the Environment, Centre for Infectious Disease Control (Clb), Bilthoven, The Netherlands. ${ }^{3}$ Central Veterinary Institute of Wageningen UR, Lelystad, The Netherlands.

\section{Received: 20 April 2016 Accepted: 23 September 2016}

\section{Published online: 04 October 2016}

\section{References}

1. Ehrenford FA. Canine ascariasis as a potential source of visceral larva migrans. Am J Trop Med Hyg. 1957;6:166-70.

2. Overgaauw PA. Aspects of Toxocara epidemiology: human toxocarosis. Crit Rev Microbiol. 1997:23(3):215-31. doi:10.3109/10408419709115137.

3. Pinelli E, Herremans T, Harms MG, Hoek D, Kortbeek LM. Toxocara and Ascaris seropositivity among patients suspected of visceral and ocular larva migrans in the Netherlands: trends from 1998 to 2009. Eur J Clin Microbiol Infect Dis. 2011:30(7):873-79. doi:10.1007/s10096-011-1170-9.

4. Fillaux J, Magnaval JF. Laboratory diagnosis of human toxocariasis. Vet Parasitol. 2013;193(4):327-36. doi:10.1016/j.vetpar.2012.12.028.

5. Parsons JC. Ascarid infections of cats and dogs. Vet Clin North Am Small Anim Pract. 1987;17(6):1307-39.

6. Glickman LT, Schantz PM. Epidemiology and pathogenesis of zoonotic toxocariasis. Epidemiol Rev. 1981;3:230-50.

7. Gamboa Ml. Effects of temperature and humidity on the development of eggs of Toxocara canis under laboratory conditions. J Helminthol. 2005; 79(4):327-31; doi:S0022149X05000454.

8. Greve JH. Age resistance to Toxocara canis in ascarid-free dogs. Am J Vet Res. 1971;32(8):1185-92.

9. Dubey JP. Patent Toxocara canis infection in ascarid-naive dogs. J Parasitol 1978;64(6):1021-23.

10. Sprent JF. Observations on the development of Toxocara canis (Werner, 1782) in the dog. Parasitology. 1958;48(1-2):184-209.

11. Maizels RM, Meghji M. Repeated patent infection of adult dogs with Toxocara canis. J Helminthol. 1984;58(4):327-33.

12. Fahrion AS, Staebler S, Deplazes P. Patent Toxocara canis infections in previously exposed and in helminth-free dogs after infection with low numbers of embryonated eggs. Vet Parasitol. 2008;152(1-2):108-15. doi:10.1016/j.vetpar.2007.11.022

13. Sager H, Moret C, Grimm F, Deplazes P, Doherr MG, Gottstein B. Coprological study on intestinal helminths in Swiss dogs: temporal aspects of anthelminthic treatment. Parasitol Res. 2006;98(4):333-38. doi:10.1007/ s00436-005-0093-8.

14. Claerebout E, Casaert S, Dalemans AC, De Wilde N, Levecke B, Vercruysse J, et al. Giardia and other intestinal parasites in different dog populations in Northern Belgium. Vet Parasitol. 2009;161(1-2):41-6. doi:10.1016/j.vetpar. 2008.11.024;10.1016/j.vetpar.2008.11.024

15. Barutzki D, Schaper R. Results of parasitological examinations of faecal samples from cats and dogs in Germany between 2003 and 2010. Parasitol Res. 2011:109 Suppl 1:S45-60. doi:10.1007/s00436-011-2402-8.

16. Nijsse R, Ploeger HW, Wagenaar JA, Mughini-Gras L. Toxocara canis in household dogs: prevalence, risk factors and owners' attitude towards deworming. Parasitol Res. 2014; doi:10.1007/s00436-014-4218-9

17. ESCCAP. Worm Control in Dogs and Cats. September 2010. http://www. esccap.org/uploads/docs/nkzqxmxn_esccapgl1endoguidelines.pdf

18. Nijsse R, Mughini-Gras L, Wagenaar JA, Franssen F, Ploeger HW. Environmental contamination with Toxocara eggs: a quantitative approach to estimate the relative contributions of dogs, cats and foxes, and to assess the efficacy of advised interventions in dogs. Parasit Vectors. 2015;8:397. doi:10.1186/s13071-015-1009-9.

19. Pullola T, Vierimaa J, Saari S, Virtala AM, Nikander S, Sukura A. Canine intestinal helminths in Finland: prevalence, risk factors and endoparasite control practices. Vet Parasitol. 2006;140(3-4):321-26; doi:S0304-4017(06)00240-8.

20. Gates MC, Nolan TJ. Risk factors for endoparasitism in dogs: retrospective case-control study of 6578 veterinary teaching hospital cases. J Small Anim Pract. 2009;50(12):636-40.

21. Mohamed AS, Moore GE, Glickman LT. Prevalence of intestinal nematode parasitism among pet dogs in the United States (2003-2006). J Am Vet Med Assoc. 2009;234(5):631-37. doi:10.2460/javma.234.5.631.

22. Overgaauw PA, van Zutphen L, Hoek D, Yaya FO, Roelfsema J, Pinelli E, et al. Zoonotic parasites in fecal samples and fur from dogs and cats in The Netherlands. Vet Parasitol. 2009;163(1-2):115-22. doi:10.1016/j.vetpar.2009.03.044.

23. Becker AC, Rohen M, Epe C, Schnieder T. Prevalence of endoparasites in stray and fostered dogs and cats in Northern Germany. Parasitol Res. 2012; $111(2): 849-57$. doi:10.1007/s00436-012-2909-7.

24. Fahrion AS, Schnyder M, Wichert B, Deplazes P. Toxocara eggs shed by dogs and cats and their molecular and morphometric species-specific identification: is the finding of $T$. cati eggs shed by dogs of epidemiological relevance? Vet Parasitol. 2011;177(1-2):186-9. doi:10.1016/j.vetpar.2010.11.028.

25. Nijsse R, Mughini-Gras L, Wagenaar JA, Ploeger HW. Coprophagy in dogs interferes in the diagnosis of parasitic infections by faecal examination. Vet Parasitol. 2014:204(3-4):304-9. doi:10.1016/j.vetpar.2014.05.019.

26. Franssen F, Nijsse R, Mulder J, Cremers H, Dam C, Takumi K, et al. Increase in number of helminth species from Dutch red foxes over a 35-year period. Parasit Vectors. 2014;7:166. doi:10.1186/1756-3305-7-166.

27. Zajac AM, Conboy GA. Veterinary clinical parasitology. 8th ed. Ames: Wiley-Blackwell; 2012.

28. Prentice RL, Williams BJ, Peterson AV. On the regression analysis of multivariate failure time data. Biometrika. 1981;68(2):373-79.

29. Lee ET, Wang JW. Statistical methods for survival data analysis. 3rd ed. Hoboken: John Wiley \& Sons; 2003.

30. Becker AC, Kraemer A, Epe C, Strube C. Sensitivity and efficiency of selected coproscopical methods-sedimentation, combined zinc sulfate sedimentation-flotation, and McMaster method. Parasitol Res. 2016:115(7):2581-7. doi:10.1007/s00436-016-5003-8.

31. Kirkpatrick CE. Epizootiology of endoparasitic infections in pet dogs and cats presented to a veterinary teaching hospital. Vet Parasitol. 1988;30(2): 113-24; doi:0304-4017(88)90158-6.

32. Nolan TJ, Smith G. Time series analysis of the prevalence of endoparasitic infections in cats and dogs presented to a veterinary teaching hospital. Vet Parasitol. 1995:59(2):87-96; doi:0304-4017(94)00742-U.

33. Engle ET. No seasonal breeding cycle in dogs. J Mammal. 1946;27:79-81.

34. Overgaauw PA, Okkens AC, Bevers MM, Kortbeek LM. Incidence of patent Toxocara canis infection in bitches during the oestrous cycle. Vet Q. 1998; 20(3):104-7. doi:10.1080/01652176.1998.9694850.

35. Roth LS, Faresjo A, Theodorsson E, Jensen P. Hair cortisol varies with season and lifestyle and relates to human interactions in German shepherd dogs. Sci Rep. 2016;6:19631. doi:10.1038/srep19631. 
36. Sprent JF. On the migratory behaviour of the larvae of various Ascaris species in white mice. II. Longevity of encapsulated larvae and their resistance to freezing and putrefaction. J Infectious Dis. 1953;92:114-7.

37. Taira K, Saitoh Y, Okada N, Sugiyama H, Kapel CM. Tolerance to low temperatures of Toxocara cati larvae in chicken muscle tissue. Vet Parasitol. 2012;189(2-4):383-6. doi:10.1016/j.vetpar.2012.04.037.

38. Taira K, Snabel V, Okada N, Saitoh Y. Effect of low temperatures on the infectivity of Toxocara cati larvae parasitized in mouse tissues. Helmintologia. 2013;50(2):83-6.

39. Casella S, Fazio F, Russo C, Giudice E, Piccione G. Acute phase proteins response in hunting dogs. J Vet Diagn Invest. 2013;25(5):577-80. doi:10.1177/1040638713495851

Submit your next manuscript to BioMed Central and we will help you at every step:

- We accept pre-submission inquiries

- Our selector tool helps you to find the most relevant journal

- We provide round the clock customer support

- Convenient online submission

- Thorough peer review

- Inclusion in PubMed and all major indexing services

- Maximum visibility for your research

Submit your manuscript at www.biomedcentral.com/submit
Biomed Central 